\title{
Modificações do método gravimétrico não enzimático para determinar fibra alimentar solúvel e insolúvel em frutos
}

\section{Modifications in the gravimetric non enzymatic method for determination of soluble and insoluble dietary fiber in fruits}

Nonete Barbosa GUERRA ${ }^{1}$

Paulo Roberto de Barros Salomão DAVID ${ }^{1}$

Débora Dias de $\mathrm{MELO}^{2}$

Adriana Barbosa Buarque VASCONCELOS 3

Mônica Rabelo Maciel GUERRA ${ }^{3}$

\section{R E S U M O}

\section{Objetivos}

Modificações do tratamento da amostra e da seqüência operacional do método gravimétrico não enzimático foram realizadas, com vistas a simplificá-lo e permitir a quantificação das frações solúvel e insolúvel da fibra alimentar total.

\section{Métodos}

A influência da liofilização foi inicialmente avaliada em amostras de goiaba e, posteriormente, em acerola, manga, pinha, sapoti e uva. As modificações inerentes à seqüência metodológica foram testadas nos referidos frutos e em abacaxi, caju, maracujá e morango.

\section{Resultados}

As médias de fibra alimentar total obtidas para goiaba liofilizada e não liofilizada: $10,47 \% \pm 1,15$ e $10,53 \% \pm 0,88$, respectivamente, demonstram: boa reprodutibilidade do método e inexistência de diferenças significativas entre os tratamentos aplicados à goiaba e demais frutos. Diferentemente do constatado na

\footnotetext{
${ }^{1}$ Departamento de Nutrição, Universidade Federal de Pernambuco. Rua dos Médicos, s/n, Campus Universitário, 50670-901, Recife, PE, Brasil. Correspondência para/Correspondence to: N.B. GUERRA. E-mail: leaal@nutricao.ufpe.br

2 Graduação em Nutrição, Universidade Federal de Pernambuco, Brasil. Bolsista PIBIC/CN Pq/UFPE.

${ }^{3}$ Graduação em Ciências Biológicas, Universidade Federal de Pernambuco, Brasil. Bolsista PIBIC/CNPq/UFPE.
} 
determinação da Fibra Alimentar Insolúvel, cujo percentual variou de 99,6\% para o caju a 54,0\% para a acerola, os dados gerados para a fibra alimentar solúvel foram desconsiderados, dada a dispersibilidade apresentada.

\section{Conclusão}

Estes resultados evidenciam que as amostras prescindem da liofilização e que a quantificação da Fibra Alimentar Solúvel requer ajustes na etapa de precipitação.

Termos de indexão: fibra alimentar, método gravimétrico não inzimático, frutas.

\section{A B S T R A C T}

\section{Objetives}

Modifications in the treatment of samples and operational sequence of the gravimetric non enzymatic method were carried out in order to simplify it and allow the quantification of soluble and insoluble quantities of the total dietary fiber.

\section{Methods}

Lyophilization influence was first evaluated in guava samples and afterward in samples of acerola, mango, sugar apples, sapodilla and grapes. The mentioned fruits and also pineapple, cashew, passion fruit and strawberry were tested for the modifications intrinsic in the methodological sequence.

\section{Results}

The average total dietary fiber for guava, both lyophilized and non-lyophilized, were: $10.50 \% \pm 0.97$ and $10.53 \% \pm 0.88$, respectively, revealing good method reproducibility and no significant differences among the treatments applied to guava and other fruits. Differently from what was found in the determination of the insoluble dietary fiber, which had a variation of $99.6 \%$ for cashew and $54.0 \%$ for acerola, the data generated for soluble dietary fiber were disregarded, due to the dispersiveness found.

\section{Conclusion}

Those results show that the samples do not depend on lyophilization and that the quantification of soluble dietary fiber needs further adjustments of the precipitation stage.

Index terms: dietary fiber, gravimetric non enzymatic method, fruits.

\section{N T RO D U Ç Ã O}

O consumo de alimentos ricos em fibra alimentar é essencial para manter a saúde e reduzir os riscos de diversas doenças, conforme pesquisas realizadas por alguns autores ${ }^{1,2,3}$.

Não obstante todas as evidências, constatase que a maioria das tabelas de composição de alimentos, utilizadas pelos profissionais de saúde e de Ciência e Tecnologia de Alimentos, se limitam a informar o conteúdo de fibra bruta, restringindo, portanto, a aplicação de suas propriedades funcionais.

Em geral, a determinação da fibra alimentar é realizada por método gravimétrico, que requer um tratamento prévio da amostra através de combinações de enzimas e soluções tampões em diferentes níveis de $\mathrm{pH}$ e temperaturas, para completa remoção do amido e parcial remoção da proteína, o que pode conduzir a perdas do material ensaiado. Em 1992, Li \& Cardozo ${ }^{4}$, considerando a reduzida concentração 
de amido $(\leq 2,0 \%)$ nos frutos e hortaliças, propuseram um procedimento simples, no qual as incubações enzimáticas são suprimidas e a suspensão das amostras é obtida pela utilização seqüencial de água e etanol, para eliminar os componentes solúveis ${ }^{5}$. Após a comprovação de sua eficácia, esta metodologia foi adotada em ação final pela Association of Oficial Analytical Chemist (AOAC), em 1998, para quantificar a Fibra Alimentar Total (FAT) ${ }^{6}$ neste grupo de alimentos. Outro aspecto importante, no entanto, permanecia indeterminado. A participação da fração de Fibra Alimentar Solúvel (FAS) (substâncias pécticas, gomas, hemiceluloses e ß glucanas) e de Fibra Alimentar Insolúvel (FAI) (a maioria hemiceluloses, celulose e lignina), cuja diferenciação é baseada na solubilidade, explica a diversidade das funções por elas exercidas.

Considerando a importância de conhecer o real potencial desse grupo de alimentos na redução do risco de doenças crônicas não transmissíveis, foi implementado este trabalho, com vistas a adequar o método desenvolvido por Li \& Cardozo 4 para quantificar os percentuais das frações solúveis e insolúveis da fibra alimentar, em frutos tropicais.

\section{MATERIALE MÉTODOS}

As amostras foram constituídas pela parte comestível de: abacaxi (Annanas comosus (L) Merril), acerola (Malpighia punicifolia), caju (Anarcadium occidentale, L.), goiaba (Psidium guajava, L.), manga (Mangífera indica, L.), maracujá (Passiflora edulis, Sims.), morango (Fragaria vesca, L.), pinha (Annona squamosa, Lin.), sapoti (M anilkara zapota (L.) Van Royeu) e uva roxa (Vitis vinífera, L. ), cujos frutos, in natura, foram adquiridos, aleatoriamente, nas condições em que são comercializados na cidade do Recife, PE. Os experimentos foram realizados no Laboratório de Experimentação e Análise de Alimentos (LEAAL) do Departamento de Nutrição da Universidade Federal de Pernambuco.

\section{Experimento 1: Modificação do tratamento das amostras}

A parte comestível da goiaba, incluindo cascas e sementes, após homogeneização, foi subdividida em dois grupos: A) liofilizada conforme o método de Li \& $\mathrm{Cardozo}^{7}$ e B) não liofilizada (amostra-teste). Nesta, a tomada de amostra in natura foi realizada tendo por base o resíduo seco de cada fruto estudado (AOAC, 920.151). Ambos os grupos foram, a seguir, submetidos aos procedimentos descritos pelo método acima referido, para determinação da FAT (Figura 1). A precisão da modificação foi avaliada considerando a reprodutibilidade dos dados em função das características da dispersão dos resultados obtidos e através da determinação dos Coeficientes de Variação $(\mathrm{CV})^{9}$. Em seguida, estes tratamentos foram aplicados à acerola, manga, sapoti, uva roxa e pinha e os resultados submetidos ao teste " $t$ " de Student, para comparação entre as médias.

\section{Experimento 2: Adequação da seqüência operacional do método, com vistas a propiciar a determinação das frações solúveis e insolúveis}

O esquema analítico de Li \& Cardozo $^{7}$ foi modificado, considerando a proposta de Prosky et al. ${ }^{10}$ (Figura 2), para a mensuração das frações insolúvel e solúvel. Para determinação da FAI, a filtração foi procedida imediatamente após a solubilização dos compostos solúveis, sendo o filtrado recuperado para quantificação da FAS, antes da lavagem do resíduo do cadinho, com etanol e acetona, prosseguindo conforme Figura 1, enquanto a FAS foi obtida a partir da precipitação dos integrantes da fração solúvel, do filtrado recuperado, seguida de nova filtração e demais etapas descritas na Figura 1. Estas modificações foram testadas nos frutos anteriormente citados e ainda em abacaxi, caju, maracujá e morango. 


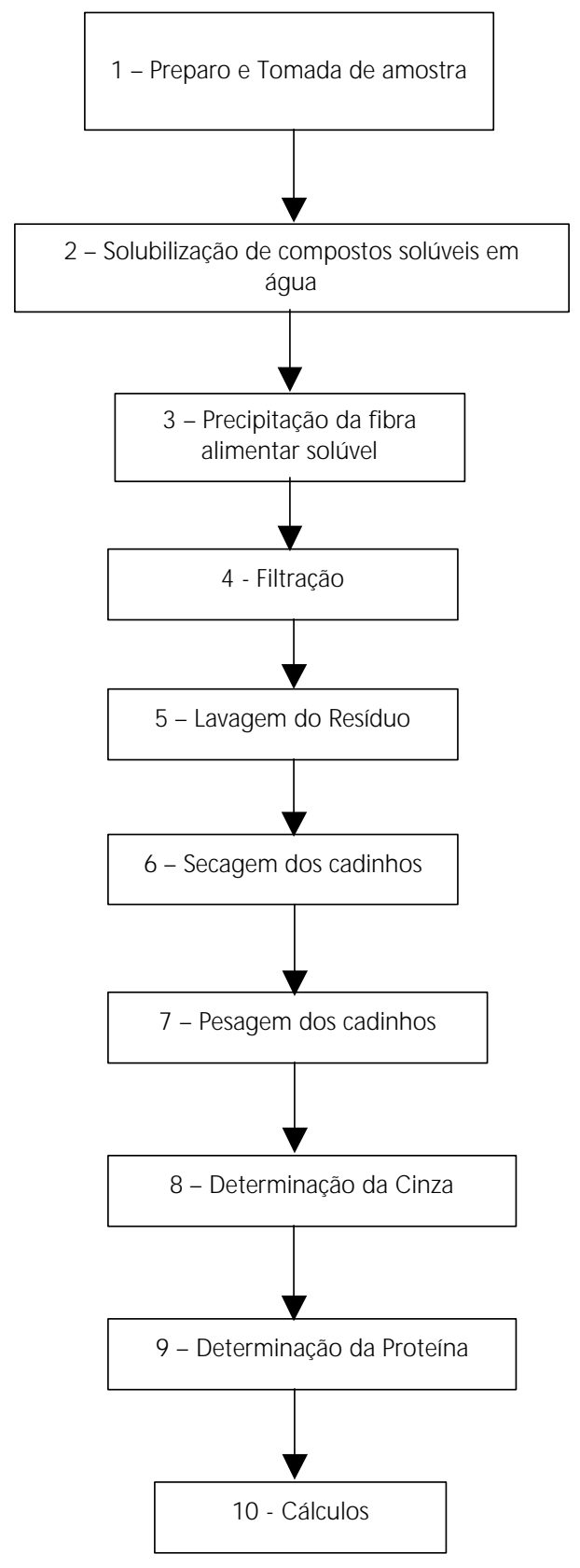

Homogeneização da parte comestível após liofilização ou não Pesar 2x500mg (Pa) e adicionar 20mL/água deionizada para completar $25 \mathrm{~mL}$ Misturar até dissolução completa da amostra.

Cobrir o Becker e deixar por $90 \mathrm{~min}$ sob agitação em banho Maria a $37^{\circ} \mathrm{C}$.

Adicionar $100 \mathrm{~mL}$ de etanol a $95 \%$, aquecido a $65^{\circ} \mathrm{C}$ e deixar por $1 \mathrm{~h}$ em agitação.

Esfriar por $90 \mathrm{~min}$ à temperatura ambiente $(25 \pm 2 \stackrel{\circ}{ } \mathrm{C})$.

Sob vácuo em cadinho de vidro tipo Gooch.

50mL/ASTM 40-60 C/Pirex №32940-50C contendo.

$500 \mathrm{mg}$ de Celite - Secar a 105C/noite.

Em $2 \times 20 \mathrm{~mL}$ de etanol $78 \%(v / v)$ e $1 \times 10 \mathrm{~mL}$ etanol $95 \%$

$\mathrm{x} 10 \mathrm{~mL}$ de acetona.

Em estufa a $105^{\circ} \mathrm{C}$, por uma noite.

Esfriar por 2h, em dessecador.

Resíduo Total $(\mathrm{RT})=(\mathrm{Pr})$.

Mufla $6 \mathrm{~h}$ a $525^{\circ} \mathrm{C}, \mathrm{AOAC}-93022$ (A).

Homogeneizar o conteúdo de todo o cadinho.

Secar em estufa a $105^{\circ} \mathrm{C}$ por uma hora.

Determinar a proteína $(\mathrm{N} \times 6,25)$ :

Kjeldhal AOAC-96052 (P).

TDF\% $=100 \times(\operatorname{Pr}-(\mathrm{P}+\mathrm{A}) / 100 \times \mathrm{Pr}) / \mathrm{Pa} ;$

$\mathrm{Pr}=\mathrm{mg}$ resíduo; $\mathrm{P}=\%$ de Proteína no resíduo;

$\mathrm{A}=\%$ de cinza no resíduo e $\mathrm{Pa}=\mathrm{mg}$ amostra.

Figura 1. Determinação de Fibra Alimentar Total pelo Método Gravimétrico Não Enzimático (Li \& Cardozo). AOAC- 99321.

\section{RESULTA DOS E DISCUSSÃ O}

A proposta referente à modificação do preparo das amostras foi, inicialmente, aplicada à goiaba, através da realização de 10 ensaios analíticos, em duplicata (Tabela 1).

Avaliando a precisão dos dados gerados nas condições em que foram obtidos (dois operadores, dois tratamentos de amostras), verifica-se que, além da inexistência de diferenças significativas ( $p>0,05)$, os mesmos se distribuíram no intervalo de dois desvios-padrão, em torno da média, considerada aceitável como expressão de variação intralaboratorial.

Os Coeficientes de Variação (CV), 10,98\% e $8,35 \%$, determinados para as amostras 
liofilizadas e não liofilizadas, respectivamente, demonstram uma satisfatória reprodutibilidade dos dados, principalmente se considerados os fatores intrínsecos e extrínsecos inerentes ao fruto, (inexistência de classificação de cultivares e estabelecimento do ponto ótimo de colheita) e ao método (possibilidade de perda de material da amostra nas etapas de filtração e lavagem do resíduo durante a análise), enquadrando-se, portanto, dentro do esperado para materiais químicos e biológicos.

Os referidos tratamentos foram, a seguir, aplicados a outros frutos tropicais, cujos resultados (Tabela 2), ratificam a eficácia das modificações.
O estabelecimento de comparações entre os resultados desta pesquisa e aqueles gerados por outros autores é dificultado pela carência de informações sobre FAT, em frutos tropicais, obtidas por metodologia similar. Através do método Fibra Detergente Neutro, Mendez et al. ${ }^{11}$ obtiveram valores similares aos desta pesquisa para abacaxi, acerola, caju e uva; superiores para maracujá e inferiores para pinha, manga e goiaba. Estas divergências podem ser devidas a limitações do método, quando empregado em alimentos com quantidades apreciáveis de pectina ${ }^{12}$, a exemplo da goiaba, pinha e manga e/ou, segundo Guerra ${ }^{13}$, relacionadas à amostragem (parte do fruto

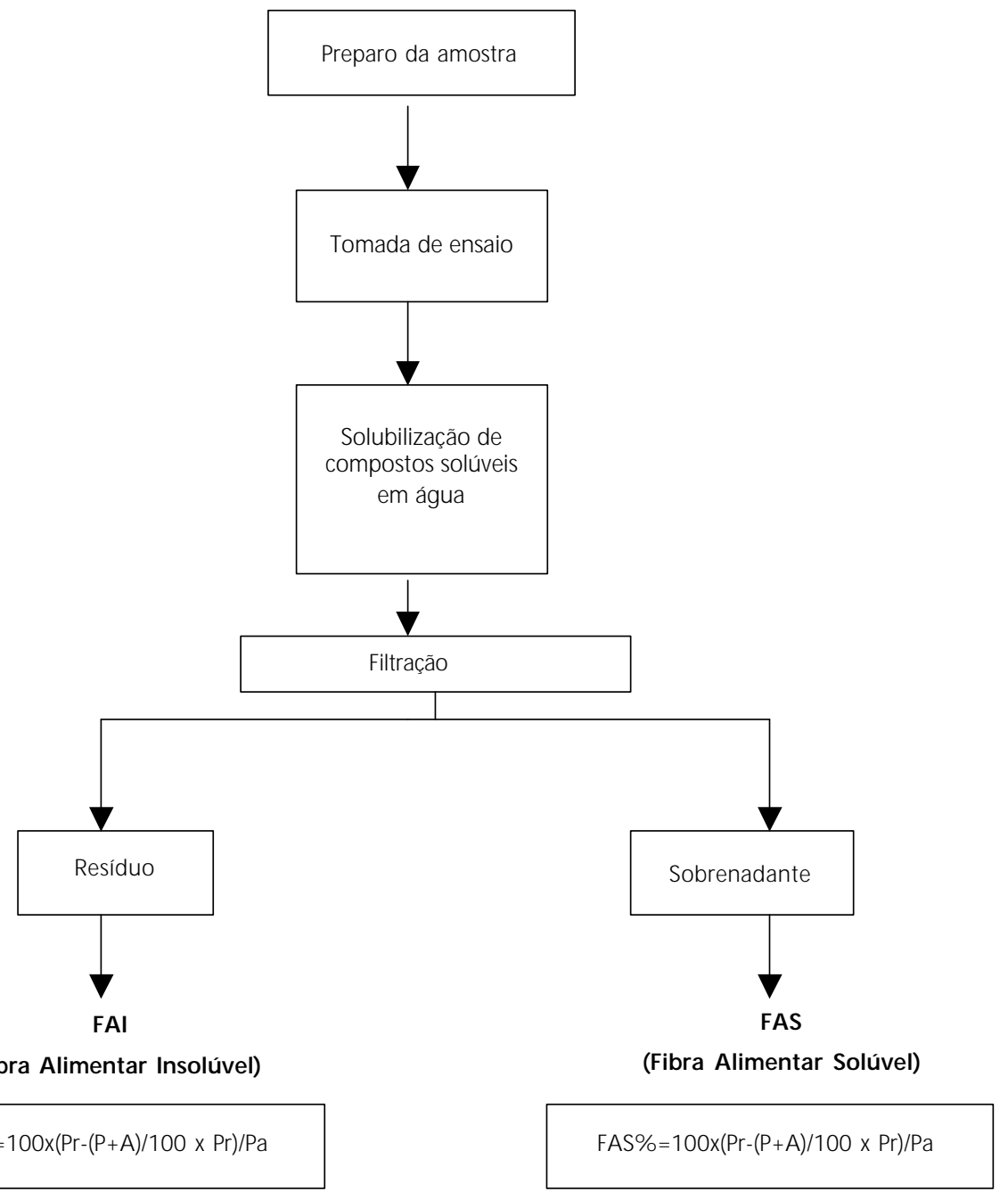

Figura 2. Esquema analítico para determinação das Frações Insolúvel e Solúvel da Fibra Alimentar pelo Método Gravimétrico Não Enzimático, adaptado por Guerra ${ }^{13}$. 
Tabela 1. Porcentagem de Fibra Alimentar Total (FAT) em goiaba liofilizada e não liofilizada.

\begin{tabular}{|c|c|c|c|}
\hline \multicolumn{2}{|c|}{ Liofilizadas } & \multicolumn{2}{|c|}{ Não Liofilizadas } \\
\hline Amostras & FAT $(\%)$ & Amostras (n) & FAT $(\%)$ \\
\hline 1 & 11,80 & 6 & 9,12 \\
\hline 2 & 10,30 & 7 & 10,45 \\
\hline 3 & 11,38 & 8 & 11,00 \\
\hline 4 & 9,98 & 9 & 11,50 \\
\hline 5 & 8,90 & 10 & 10,61 \\
\hline Média & 10,47 & Média & 10,53 \\
\hline Desvio-Padrão & 1,15 & Desvio-Padrão & 0,88 \\
\hline CV\% & 10,98 & $\mathrm{CV} \%$ & 8,35 \\
\hline
\end{tabular}

Tabela 2. Efeito do tratamento da amostra sobre o percentual de Fibra Alimentar Total, em frutos tropicais.

\begin{tabular}{lcc}
\hline Frutas & Liofilizadas* $^{*}$ & Não Liofilizadas* \\
\hline Acerola & $2,49^{\mathrm{a}}$ & $2,32^{\mathrm{a}}$ \\
Goiaba & $10,61^{\mathrm{a}}$ & $11,38^{\mathrm{a}}$ \\
Manga & $3,27^{\mathrm{a}}$ & $3,34^{\mathrm{a}}$ \\
Pinha & $5,62^{\mathrm{a}}$ & $6,43^{\mathrm{a}}$ \\
Sapoti & $9,98^{\mathrm{a}}$ & $8,63^{\mathrm{a}}$ \\
Uva Roxa & $2,27^{\mathrm{a}}$ & $2,18^{\mathrm{a}}$ \\
\hline
\end{tabular}

Nota: Na mesma linha, letras iguais não diferem estatisticamente ao nível de $5 \%$. ${ }^{(*)}$ Média obtida de, no máximo, 3 ensaios.

analisada, cultivar utilizada e/ou estádio de maturação dos frutos). Uma análise dos percentuais máximos e mínimos obtidos no decorrer desta pesquisa, independente do tratamento aplicado, comprova a influência desses fatores para goiaba, pinha e uva roxa: 8,90-11,80; 4,99 - 7,20 e 1,93 - 2,27, respectivamente.
Com relação às respostas obtidas em função das modificações introduzidas na seqüência operacional do método (experimento 2 ), foram observados problemas referentes à precipitação da FAS, provavelmente decorrentes da heterogeneidade e da reduzida concentração das substâncias integrantes desta fração, conforme já evidenciado por Prosky et al. ${ }^{10}$, que dificultaram a precipitação da mesma. Este problema, ao comprometer a precisão analítica desejada, justifica a utilização do cálculo, por diferença, para quantificar a referida fração.

Ao comparar os resultados explicitados (Tabela 3) com os apresentados por Menezes \& Lajolo ${ }^{14}$, foram constatadas similaridades, no tocante ao abacaxi $(1,36 \%)$, manga $(3,30 \%)$ e uva roxa $(1,78 \%)$ e considerável diferença em relação à goiaba (4,95\% ). Esta discrepância deve

Tabela 3. Utilização do Método Gravimétrico Não Enzimático modificado na determinação da Fibra Alimentar Total, Insolúvel e Solúvel, em frutos tropicais.

\begin{tabular}{lccc}
\hline Amostras Frutos & Fibra Alimentar Total $(\mathrm{g} \%)$ & Fibra Insolúvel $(\mathrm{g} \%)$ & Fibra Solúvel $(\mathrm{g} \%)$ \\
\hline Abacaxi & 1,34 & 1,14 & 0,20 \\
Acerola & 2,22 & 1,21 & 1,01 \\
Caju & 2,66 & 2,65 & 0,01 \\
Goiaba & 11,5 & 9,96 & 1,54 \\
Manga & 3,34 & 2,08 & 1,26 \\
Maracujá & 0,58 & 0,53 & 0,05 \\
Morango & 1,99 & 1,73 & 0,26 \\
Pinha & 5,44 & 4,59 & 0,85 \\
Sapoti & 8,63 & 7,29 & 1,34 \\
Uva Roxa & 1,96 & 1,70 & 0,26 \\
\hline
\end{tabular}

(*) Calculada por diferença (Fibra alimentar total - Fibra Insolúvel = Fibra solúvel). 
ser decorrente da amostragem utilizada nesta pesquisa que incluiu cascas e sementes, em consideração ao hábito de consumo local do referido fruto. Uma considerável variabilidade foi observada, entre os frutos estudados, quanto ao teor de FAT, que se apresenta mais elevado na goiaba, sapoti e pinha, corroborando os achados de Salgado et al. ${ }^{15}$.

Embora tenha sido ratificada a predominância da fração insolúvel: 99,6\% , 86,6\% , 84,4\% e $84,3 \%$ da FAT, no caju, goiaba, sapoti e pinha, respectivamente, destaca-se a participação da fibra solúvel, na manga e acerola, cuja relação com a insolúvel é de aproximadamente $38: 62 \%$ e 46:54\%. Considerando as recomendações do Food and Drug Administration (FDA) para uma dieta equilibrada: $70 \%-75 \%$ de fibra insolúvel para 25\% -30\% de solúvel ${ }^{3}$, verifica-se que a relação entre as frações de fibra da manga e da acerola encontra-se próxima ao ideal, diferenciando-os dos demais frutos estudados.

Estas informações são relevantes, do ponto de vista prático, na elaboração de dietas, face aos possíveis efeitos de cada uma das frações. Os componentes da FAI, por exemplo, são dotados de propriedades higroscópicas, que Ihes conferem a habilidade de reter água no trato gastrintestinal, aumentando o volume das fezes e, conseqüentemente, reduzindo o tempo de trânsito, o que permite corrigir a constipação, prevenindo, assim, o aparecimento de alterações patológicas no intestino, enquanto as substâncias integrantes da FAS apresentam um efeito diferencial sobre os níveis de colesterol no plasma ${ }^{1,2,3,16-18}$.

Do exposto, depreende-se a importância de gerar e divulgar informações sobre as frações da fibra, através de tabela de composição e da rotulagem de alimentos, com vistas a orientar 0 consumidor na seleção de alimentos para compor uma dieta rica em fibra e auferir seus benéficos efeitos sobre a saúde.

\section{O N C L U S Ã O}

Os resultados obtidos, nas condições em que a pesquisa foi realizada, permitem concluir que a etapa de liofilização não é imprescindível à determinação da fibra alimentar total, bem como de suas frações, pelo método gravimétrico não enzimático e que as modificações introduzidas na seqüência operacional deste método, embora eficazes na determinação da Fração Insolúvel (FAI), necessitam ajustes para a quantificação da Fração Solúvel (FAZ) da Fibra Alimentar Total, em frutos tropicais.

\section{REFERÊ N C I A S}

1. Schneeman BO, Tietyen J. Dietary fiber. In: Shils ME, Olson JA, Shike M. M odern nutrition in health and disease. 8th ed. Philadelphia: Lea \& Febiger; 1996. v.1

2. Schimdt MK, Labuza TP. Essential of functional foods. Gaithersburg: Aspem Publication; 2000. $395 p$.

3. Marquez LR. A fibra terapêutica. São Paulo: CRF Propaganda; 2001. 175p.

4. Li BW, Cardozo MS. Nonenzimatic-gravimetric determination of total dietary fiber in fruits and vegetables. J AOAC Int 1992; 75(2):372-4.

5. Lajolo FM , Saura-Calixto F, Penna EW, M enezes EW. Fibra dietética em Iberoamerica: tecnologia y salud: obtención, caracterización, efecto fisiológico y aplicación em alimentos. São Paulo: Varela; 2001. $469 p$.

6. Filisetti TMCC. Fibra alimentar insolúvel, solúvel e total em alimentos brasileiros. Rev Farmácia e Bioquímica da Universidade de São Paulo 1991; 27(1):83-97.

7. Li BW, Cardozo MS. Nonenzymatic-gravimetric determination of dietary fiber in fruits and vegetables. J AOAC Int 1994; 77(3):687-9.

8. Association of Official Analytical Chemists. Official methods of analysis. 16th ed. Washington; 1998. v.la, lla.

9. Bredolan G. Validação de métodos cromatográficos. Pedreira: Expolabor e Isolabor Consultoria e Treinamento; 2000 (Seminário). 
10. Prosky L, Asp NG, Scew eizer TF. Determination of insoluble, soluble and total fiber in food products: interlaboratory study. J Assoc Off Anal Chem 1988; 71(5):1017-23.

11. Mendez M HM , Dewi SCN, Rodrigues M CR. Tabela de composição de alimento. Niterói: Universidade Federal Fluminense; 1995. 41p.

12. Slavin JL. Dietary fiber: classification, chemical analysis and food sources. J Am Diet Assoc 1987; 87(9):1164-71.

13. Guerra NB. Constituintes da fibra alimentar em frutos tropicais in natura e derivados. Relatório ao CNPQ (Pesquisa). Recife: Departamento de Nutrição Universidade Federal de Pernambuco; 1999. $19 p$.

14. Menezes EW, Lajolo FM. Contenido en fibra dietética y almidon resistente in alimentos y productos Ibero-americanos. São Paulo: DOCUPRINT; 2000. 121p.

15. Salgado SM, Guerra NB, M elo Filho AB. Polpa de frutos congelada: efeito do processamento sobre o conteúdo de fibra alimentar. Rev Nutr 1999; 12(3):303-8.

16. Schneeman BO. Dietary fiber physical and chemical proper pies, methods of analysis and physiological effects. Food Technol 1986; 40(2):104-10.

17. Rispin CM, Keenen JM, Jacobs DR, Elmeme PJ. Oat products on lipid lowering a meta analysis. J Am Med Assoc 1992; 267:3317-27.

18. Gallaher DD, Schneeman BO. Fibra alimentaria. In: Ziegler EE, Filer Jr LJ. Conocimientos actuales sobre nutricion. 7.ed. Washington: OMS; 1997:95-105.

Recebido para publicação em 31 de setembro de 2001 e aceito em 28 de abril de 2003. 